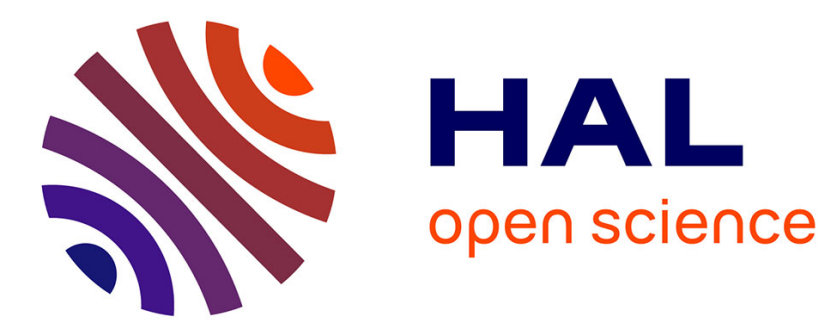

\title{
Nanoporous beta-PVDF membranes with selectively functionalized pores
}

Olivia Cuscito, M.C. Clochard, Stéphane Esnouf, Natacha Betz, Didier Lairez

\section{To cite this version:}

Olivia Cuscito, M.C. Clochard, Stéphane Esnouf, Natacha Betz, Didier Lairez. Nanoporous betaPVDF membranes with selectively functionalized pores. Nuclear Instruments and Methods in Physics Research Section B: Beam Interactions with Materials and Atoms, 2007, 265, pp.309-313. 10.1016/j.nimb.2007.08.089 . hal-00590410

\section{HAL Id: hal-00590410 https://hal.science/hal-00590410}

Submitted on 11 Mar 2015

HAL is a multi-disciplinary open access archive for the deposit and dissemination of scientific research documents, whether they are published or not. The documents may come from teaching and research institutions in France or abroad, or from public or private research centers.
L'archive ouverte pluridisciplinaire HAL, est destinée au dépôt et à la diffusion de documents scientifiques de niveau recherche, publiés ou non, émanant des établissements d'enseignement et de recherche français ou étrangers, des laboratoires publics ou privés. 


\title{
Nanoporous $\beta$-PVDF membranes with selectively functionalized pores *
}

\author{
O. Cuscito, M.-C. Clochard * , S. Esnouf, N. Betz \\ LSI, Ecole Polytechnique, CEA-DSM, CNRS, 91128 Palaiseau cedex, France \\ D. Lairez \\ Laboratoire Léon Brillouin, CEA-Saclay, 91191 Gif-sur-Yvette cedex, France
}

\begin{abstract}
Poly (vinylidene fluoride) ( $\beta$-PVDF) nanoporous membranes are obtained by heavy ion irradiation and track etching leading to cylindrical pores. Pores diameter measured by Scanning Electron Microscopy and Small Angle Neutron Scattering lies in the $20-50 \mathrm{~nm}$ range. Electron Paramagnetic Resonance study gives evidence that radicals still remains in PVDF membrane after track-etching. These radicals allows acrylic acid polymerization to be initiated onto membrane. So radiografted and functionalized membranes are characterized using Infrared Spectroscopy, weighing measurements and Energy Dispersive Spectroscopy. Finally, radiografted poly(acrylic-acid) (PAA) has been selectively labeled by fluorophores and imaged by Confocal Laser Scanning Microscopy. Images show the localisation of PAA specifically inside nanopores.
\end{abstract}

Key words: $\beta$-PVDF Membranes, Swift heavy ions, Nanopores, Poly (acrylic acid) PACS: 81.07.-b, 82.45.Mp, 61.80.Lj

\section{Introduction}

Swift heavy ion irradiation of polymers offers many applications depending on ion species, specific energy of ion, particle flux and fluence. Nanoporous membranes is an application field of increasing interest [1]. With the aim of preparing such membranes, irradiation of poly(vinylidene fluoride) (PVDF) in $\beta$-phase is particularly interesting due to: 1 )biocompatibility; 2)excellent chemical properties of PVDF. However, PVDF hydrophobicity restricts possible applications in aqueous media such as those for biological applications. Different methods can be

\footnotetext{
* Supported by the french Ministry of Scientific Research (ACI "nanoscience" 2003, grant $\mathrm{n}^{\circ} 120$ ).

* Corresponding author:

marie-claude.clochard@polytechnique.edu
}

applied to modify membranes surface. Among these methods, radiografting polymerization consists in either creating active sites by $\gamma$-irradiation $[2,3]$, or plasma treatment [4], or electron irradiation [5] and then initiate polymerization with hydrophilic groups. In different works, Mazzei et al. displayed polymerization should be initiated from active sites remaining after etching.

In this paper, we prove by Electron Paramagnetic Resonance study that in the case of PVDF membranes, the radicals formed all along ion-tracks are very stable and remain significantly present around pore-walls after track etching. These radicals allow us to initiate acrylic acid polymerization and specific pore functionalization. This latter feature has been evidenced by Confocal Scanning Laser Microscopy (CSLM). 


\section{Materials and methods}

Materials: Poly(vinylidene fluoride) (PVDF) films of $9 \mu \mathrm{m}$ thickness were kindly provided by Solvay, Belgium. Toluene, potassium hydroxide, potassium permanganate, potassium disulfite, acrylic acid (AA), Mohr's salt $\left(\left(\mathrm{NH}_{4}\right)_{2} \mathrm{Fe}\left(\mathrm{SO}_{4}\right)_{2}\right.$ $\left.6 \mathrm{H}_{2} \mathrm{O}\right)$, sulphuric acid, EDC $\left(\mathrm{C}_{8} \mathrm{H}_{17} \mathrm{~N}_{3} \mathrm{HCl}\right)$, phosphate buffer saline (PBS), tBuOK $\left(\mathrm{C}_{4} \mathrm{H}_{9} \mathrm{OK} 95 \%\right)$ were purchased from Sigma-Aldrich. Alexa Fluor ${ }^{\circledR}$ 488 hydrazide $\left(\mathrm{C}_{21} \mathrm{H}_{15} \mathrm{~N}_{4} \mathrm{NaO}_{10} \mathrm{~S}_{2}\right)$ was purchased from Invitrogen.

Irradiations: Before irradiations, $\beta$-PVDF films are toluene-extracted for $24 \mathrm{~h}$. PVDF films were electron irradiated using a Van de Graaff accelerator (absorbed dose of $100 \mathrm{KGy}$, under He atmosphere). Swift heavy ion irradiations were performed at the GANIL, Caen. Films were irradiated with $\mathrm{Kr}$ ions (10.37 Mev/amu, fluence $10^{7}$ and $\left.5 \times 10^{8} \mathrm{~cm}^{-} 2\right)$ under He atmosphere. In two cases, samples were stored at $-20^{\circ} \mathrm{C}$ under $\mathrm{N}_{2}$ atmosphere until chemical etching and radiografting.

Chemical etching: $\beta$-PVDF irradiated films were chemically etched using permanganate solution $(0.25 \mathrm{M})$ in a highly alkaline medium $(\mathrm{KOH}, 10 \mathrm{M})$ at $65^{\circ} \mathrm{C}$ with different etching times from 0.5 to $3 \mathrm{~h}$. Membranes obtained were washed in potassium disulfite solution (15\%) then dried at $50^{\circ} \mathrm{C}$ under vacuum.

Radiografting: $\quad \beta-\mathrm{PVDF}$ film of initial size $20 \times 20 \mathrm{~mm}^{2}$, is weighted. Film is immersed at room temperature into a radiografting solution containing acrylic acid and Mohr's salt $(0.25 \%$ w/w). After 15 min of bubbling nitrogen at room temperature, sample container is introduced in a thermostated water bath at $60^{\circ} \mathrm{C}$ for $1 \mathrm{~h}$. Film is washed with water and then Sohxlet-extracted in boiled water in order to extract free homopolymer. Once dried at $50^{\circ} \mathrm{C}$ under vacuum, the radiografting yield, $Y=$ $\left(m_{f}-m_{i}\right) / m_{i}$ is calculated with $m_{i}$ and $m_{f}$ the weight before and after radiografting, respectively.

Infra-red spectroscopy: FTIR spectra of PVDF were obtained with a Nicolet Magna-IR 750 spectrometer equipped with a DGTS detector. Measurements were performed at the Brewster's angle to eliminate interference fringes. To analyse the first micrometers of the film, spectra were recorded in an Attenuated Total Reflexion mode (ATR) using a diamond-crystal with single reflection. Spectra were collected by cumulating 32 scans at a resolution of $2 \mathrm{~cm}^{-1}$.
Electron Paramagnetic Resonance (EPR): EPR spectra were recorded at the $\mathrm{X}$ band $(9.4 \mathrm{GHz})$ on a Brüker ER-200D EPR spectrometer. $\beta$-PVDF membranes were analysed before, after etching and after annealing at to $65^{\circ} \mathrm{C}$.

Fluorescein diffusion: $\beta$-PVDF membrane separates an upstream compartment with fluorescein solution $(1 \mathrm{mM})$, from a downstream compartment with pure water. Increase of fluorescein concentration, $C$, in the downstream compartment is measured by UV spectroscopy. In both compartments, a stirring was applied. Typically, $C=C_{\infty}\left(1-e^{-t / \tau}\right)$, with $C_{\infty}$ the equilibrium concentration and $\tau$ the characteristic diffusion time of fluorescein through the membrane. Denoting $A_{m}$ the membrane area, the effective exchange area is $A=A_{m} n \pi r^{2}$, with $n$ the fluence and $r$ the pore radius. Denoting, $\bar{V}$ the harmonic mean volume of the two compartments, $\Delta x$ the thickness of the membrane and $D=(430 \pm$ $30) \times 10^{-8} \mathrm{~cm}^{2} / \mathrm{s}[6]$ the diffusion coefficient of fluorescein, the measured characteristic time writes $\tau=$ $\bar{V} \Delta x /(2 A \times D)$, allowing pore radius to be calculated.

Small Angle Neutron Scattering (SANS): Measurements were performed on PACE spectrometer at LLB (CEA-Saclay). For each measurements, 18 identical $\beta$-PVDF membranes of $1 \times 1 \mathrm{~cm}^{2}(9 \mu \mathrm{m}$ thickness, fluence $\left.5 \times 10^{8} \mathrm{~cm}^{-2}\right)$ were stacked to increase neutron scattered intensity recorded as a function of scattering vector. Data treatment was performed following ref.[7]. Scattered intensity profil is accounted for using the form factor of cylinder [8], narrow distribution of orientations and convolved by spectrometer resolution [9]. Measurements were performed for membranes obtained with different etching times: 15, 20, 25 and $35 \mathrm{~min}$, respectively.

Scanning Electron Microscopy (SEM): SEM were performed with a Philips apparatus equipped with a LaB6 tip (LMS, Ecole Polytechnique). This apparatus is coupled with PGT-Princeton Gamma Tech X-ray detector (EDS) and a PRISM Digital spectrometer. Samples were immersed within a potassium ter-butoxide solution $(50 \% \mathrm{w} / \mathrm{w})$. Purple coloration appears of intensity varying with the radiografting yield. Films were embedded in EPON resin before being cut by a Leica microtome. Then, profiles of cross-section can be imaged. Each sample is coated with $\mathrm{Au}$ layer.

Confocal Scanning Laser Microscopy (CSLM): Measurements were performed at LLB with a Leica TCS-SP2 using a Ar laser (488 nm). Samples were observed in water with a $40 \times$ dry objective of 
numerical aperture 0.85 .

\section{Results}

\subsection{Nanopores diameter}

Nanopores diameter of $\beta$-PVDF membranes was determined by SEM, SANS and fluorecein diffusion measurements for different etching time.

In Fig.1, a typical SEM image is shown for pores diameter of $30 \mathrm{~nm}$. Below this size, pores diameter becomes difficult to evaluate by SEM. Two additional limitations are inherent to this technique: 1) only surface information are obtained; 2) statistics over macroscopic length scales is difficult to achieve.

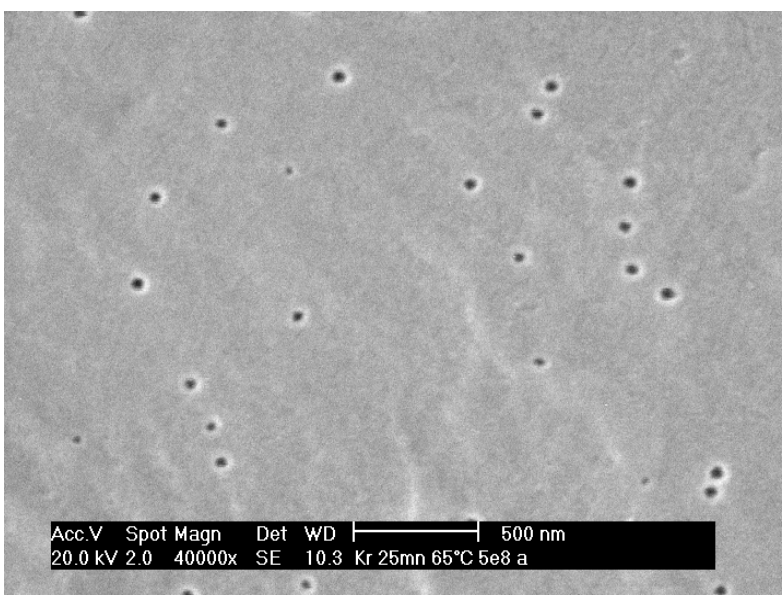

Fig. 1. SEM image of the surface of a typical track etched $\beta$-PVDF membrane: fluence $5 \times 10^{8} \mathrm{~cm}^{-2}$, etching time $25 \mathrm{~min}$.

In Fig.2, SANS spectra obtained for pores diameter between 20 and $50 \mathrm{~nm}$ are plotted as a function of scattering vector $q$. For each spectra, the position, $q^{*}$, of the maximum is proportional to the reverse diameter, $1 / \phi$. One has: $q^{*} \simeq 4 / \phi$. Pores diameter smaller than $20 \mathrm{~nm}$ should be easily measured. Compared to SEM, note that: 1) SANS provides informations concerned with the bulk of membranes; 2) SANS signal is directly an average on sample of macroscopic size.

In Fig.3, results obtained by the different methods are compared. In addition, pores diameter deduced from measurements of fluorescein diffusion through membranes are plotted. SEM results suffer from small size limitations and important error bars, whereas measurements of fluorescein diffusion show huge error bars. Actually, these latter measurements are concerned with very long diffusion time (several days). Then temperature stability and the corresponding convection problems cannot be avoided. Finally, SANS gives the best results.

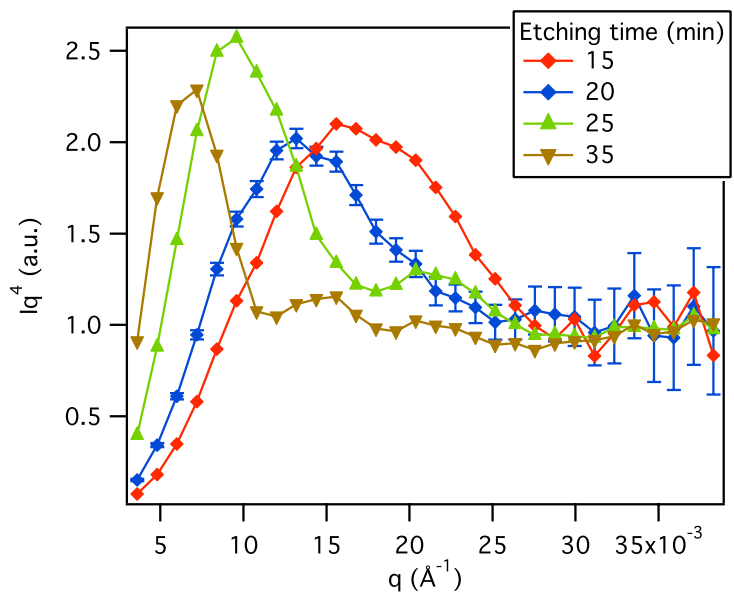

Fig. 2. Small angle neutron scattering intensity, $I$, multiplied by $q^{4}$ vs. scattering vector $q$ for $\beta$-PVDF membrane with different etching times. For increasing etching time, a shift of the maximum of the spectra to lower $q$ values indicates increasing pores diameter.

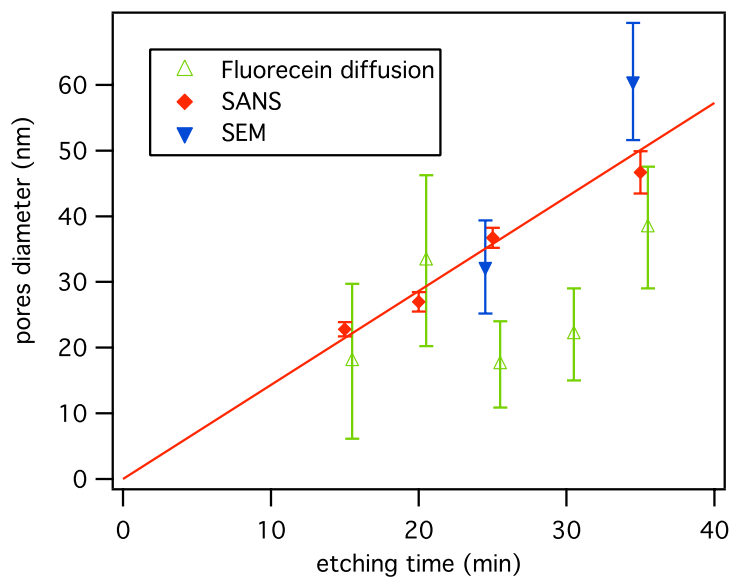

Fig. 3. Pores diameter, $\phi$, of $\beta$-PVDF membrane vs. etching time. Measurement performed by SEM, SANS and Fluorescein diffusion. Straight line is the best linear fit of SANS results: $\phi=(1.43 \pm 0.04) \times t$.

\subsection{Radicals after chemical etching}

During chemical etching at $65^{\circ} \mathrm{C}$, radicals amount decreases with time because of a loss of matter, but they are still present in the membrane after etching. 
This was evidenced by Electron Paramagnetic Resonance (EPR) (see Fig.4). We observed that approximately $20 \%$ of radicals initially present in irradiated membrane remain present after $1 \mathrm{~h}$ of etching. Note that this etching time corresponds to pores of $85 \mathrm{~nm}$ diameter (see section 3.1). The nature of these radicals is known [10,11]: alkyl radicals (in-chain $-\mathrm{CF}_{2^{-}}$ $\mathrm{C}^{\bullet} \mathrm{H}-\mathrm{CF}_{2}$ - and end-chain $-\mathrm{CF}_{2}-\mathrm{C}^{\bullet} \mathrm{H}_{2}$ ), peroxy radicals $\left(\mathrm{POO}^{\bullet}\right)$. These actives sites are able to initiate radiografting and polymerization of acrylic acid.

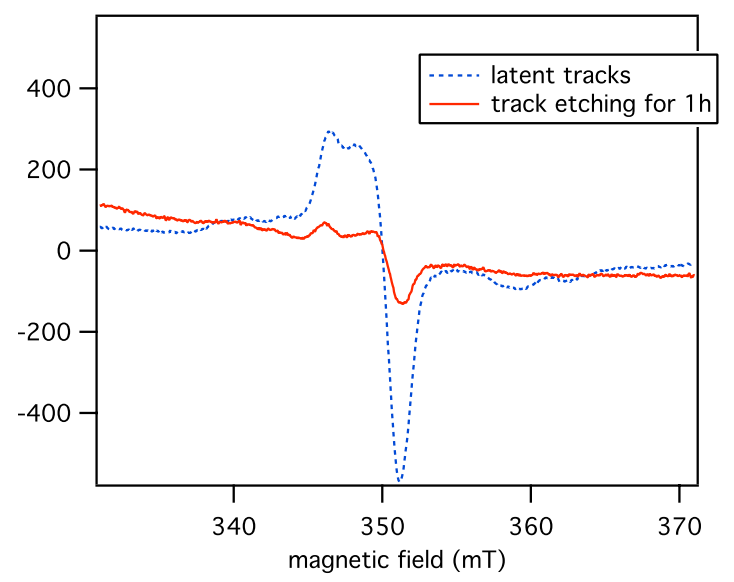

Fig. 4. Electron Paramagnetic Resonance (EPR) spectra per mass unit $\left(\mathrm{mg}^{-1}\right)$ for heavy ions irradiated film before etching (latent tracks) and after $1 \mathrm{~h}$ track etching. In this latter case, non-horizontal baseline is due to paramagnetic impurities $\left(\mathrm{KMnO}_{4}\right.$ from etching bath).

In order to assert the existence of radicals after chemical etching, samples allowing FTIR spectroscopy measurements to be performed were specially prepared: film thickness $25 \mu \mathrm{m}$, fluence $10^{9} \mathrm{~cm}^{-2}$, radiografting with $100 \%$ AA. FTIR spectra of these PVDF-g-PAA membranes show a characteristic band for the $\mathrm{O}-\mathrm{C}=\mathrm{O}$ stretching $\left(\nu=1710 \mathrm{~cm}^{-1}\right)$ related to the $\mathrm{COOH}$ groups of radiografted PAA chains (see Fig.5).

\subsection{Radiografting: pore-wall modeling by electron irradiated films}

The passage of one swift heavy ion through PVDF film comes with energy transfer. Above ion energy of the order of $0.1 \mathrm{MeV} / \mathrm{mau}$, this transfer is mainly electronic. It results in damage-zones of two species [12]:

(i) Atomic collision-cascades over distances from ion trajectory of the order of $0.01 \mu \mathrm{m}$ forms the track-core that is a highly damaged zone.

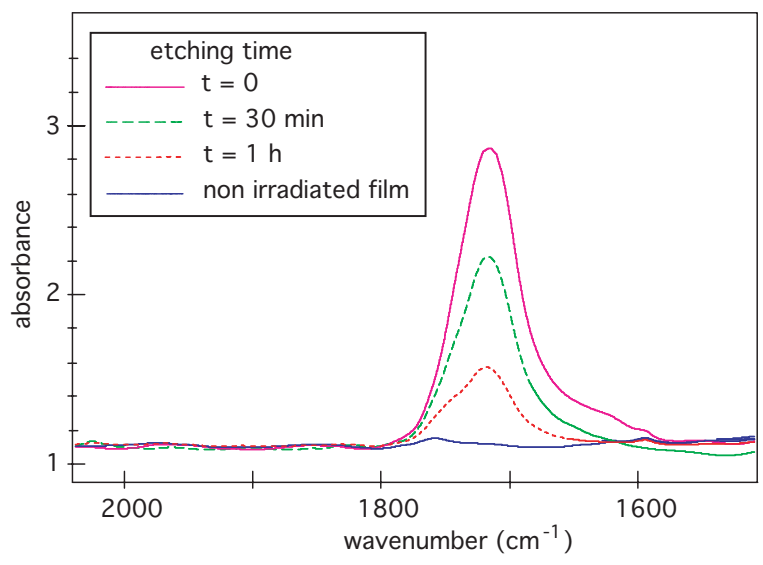

Fig. 5. FTIR spectra of swift heavy ion irradiated PVDF-gPAA for different etching times (AA concentration $100 \% \mathrm{v} / \mathrm{v}$, fluence $10^{9} \mathrm{~cm}^{-2}$, Mohr's salt $0.25 \% \mathrm{w} / \mathrm{w}$, radiografting at $60^{\circ} \mathrm{C}$ for $1 \mathrm{~h}$ ). The maximum corresponds to $\mathrm{O}-\mathrm{C}=\mathrm{O}$ stretching $\left(\nu=1710 \mathrm{~cm}^{-1}\right)$ and gives evidence for $\mathrm{COOH}$ groups of radiografted PAA chains.

(ii) Electronic collision-cascades over larger distances form the trak-shell. This zone is enlarged up to a diameter of the order of micrometer.

After track etching, only this latter damaged zone participates to the pore-wall (see Fig.6). In the case of electron irradiation, bulk and surface of the film are homogeneously irradiated leading to damages of the second species. Thus, with respect to radiografting properties, similar behavior are expected for ion irradiated films after track etching and electron irradiated films. Such modeling allows us a quantitative study of radiografting that should be difficult otherwise (the radicals amount determined by EPR after electron irradiation is 13 times higher than the one after ion irradiation at fluence of $5 \times 10^{8} \mathrm{~cm}^{-2}$ ).

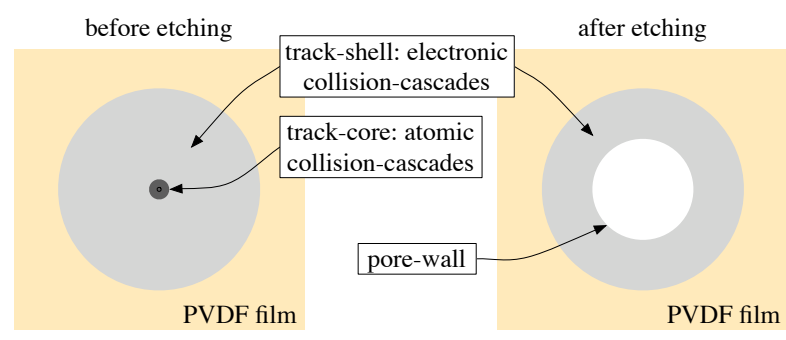

Fig. 6. Schematic representation of an ion track before and after etching. For heavy ion irradiation of PVDF film, the particular "core-shell" structure of ion track, allows us to use electron irradiated film as model of pore-wall.

Radiografting of PAA on PVDF after electron irradiation has been already studied in detail [13]. In Fig. 7 for electron and ion irradiated films, the varia- 
tion of PAA radiografting yield is plotted as a function of the AA concentration in the reaction bath. Apart from a shift of the maximum, the shape of the two curves is the same: radiografting yield linearly increases with AA concentration up to $50 \% \mathrm{v} / \mathrm{v}$; then it increases more rapidly until a maximum and finally decreases. In case of etched foils, the grafting yield displays similar curve (versus AA concentration) at a smaller scale. This behavior has been already observed for $\alpha$-PVDF. It is due to cooperative effects of solvent and monomer diffusion inside PVDF film [13].

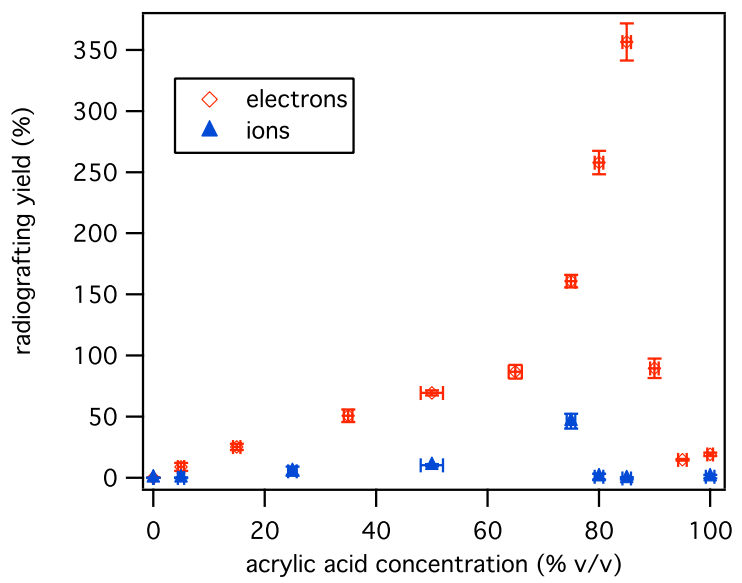

Fig. 7. Radiografting yield vs. AA concentration. Open symbols: ion irradiated film, at $100 \mathrm{kGy}$. Full symbols: Kr ion irradiated film, fluence $5 \times 10^{8} \mathrm{~cm}^{-2}$, no etching. Radiografting at $60^{\circ} \mathrm{C}$ for $1 \mathrm{~h}$.

This latter point is illustrated by Energy Dispersive Spectroscopy (EDS) study of film cross-section. Fig. 8 shows EDS profiles for $\beta$-PVDF-g-PAA films obtained after electron irradiation for acrylic acid concentration of $5 \% \mathrm{v} / \mathrm{v}$. It appears that monomers reach the inner part of the film, leading to an homogeneous radiografting. For this AA concentration, radiografted pore-walls presumably also exhibit a zone of closely interpenetrated PAA and PVDF chains (see Fig.9).

\subsection{Visualization of labelled PAA radiografted pores by $C L S M$}

Classical analysis techniques of radiografting such as those used in section 3.2, cannot be used for thin PVDF membranes and low fluence. Confocal Laser Scanning Microscopy (CLSM) was used. This technique provides three dimensions images of structures or objets labeled by fluorescent molecules

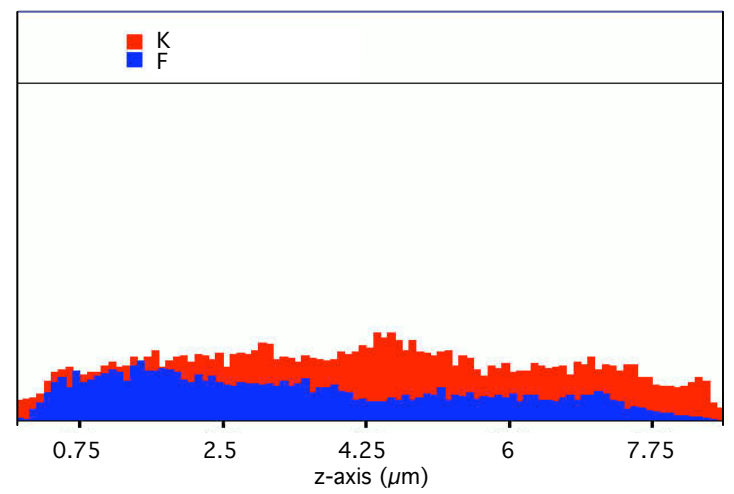

Fig. 8. Cross-section EDS profiles (red: potassium, blue: fluorine atoms) of PVDF-g-PAA films initially electron irradiated at $100 \mathrm{kGy}$, radiografted at $60^{\circ} \mathrm{C}$ for $1 \mathrm{~h}$ (AA concentration $5 \% \mathrm{v} / \mathrm{v}$, grafting yield $9 \% \mathrm{w} / \mathrm{w}$ ) with $0.25 \% \mathrm{w} / \mathrm{w}$ of Mohr's salt and treated in solution of tBuOK $(50 \% \mathrm{w} / \mathrm{w})$.

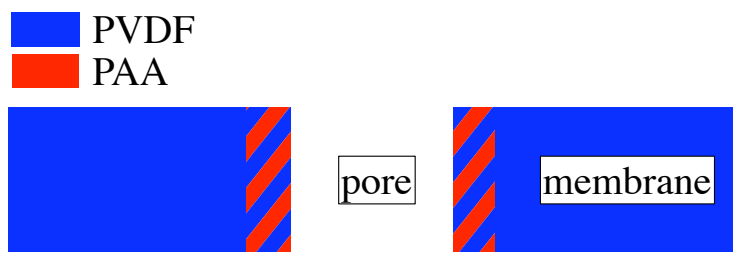

Fig. 9. Schematic representation of pore wall structure made of interpenetrated PVDF and radiografted PAA.

beforehand. Laser causes fluorophores excitation and the consecutive fluorescence of the focal volume (voxel) is measured. Three dimensional scanning of this voxel provides $3 \mathrm{D}$ images.

In our case, radiografted PAA can be labeled by Alexa Fluor ${ }^{\circledR}$ hydrazide specially designed for carboxyl group labeling. However, etching bath is oxidative and responsible for the formation of carboxyl group at the surface of film. In order to differentiate the two carboxyl group species (PAA carboxyl and oxydated PVDF carboxyl), the following method was used:

(i) After track etching and before PAA radiografting, oxydated PVDF carboxyl groups are modified with ethylenediamine $\left(\mathrm{NH}_{2}-\mathrm{CH}_{2}\right.$ $\mathrm{CH}_{2}-\mathrm{NH}_{2}$ ) leading to primary amine groups at the surface of the film.

(ii) Membrane is then radiografted with PAA. Surface amine groups can be revealed by fluorescein isothiocyanate (FITC) labeling. FITC is a fluorophore that specifically reacts with primary amine groups. The corresponding CLSM image is shown in Fig.10 top. PAA carboxyl groups are revealed by 

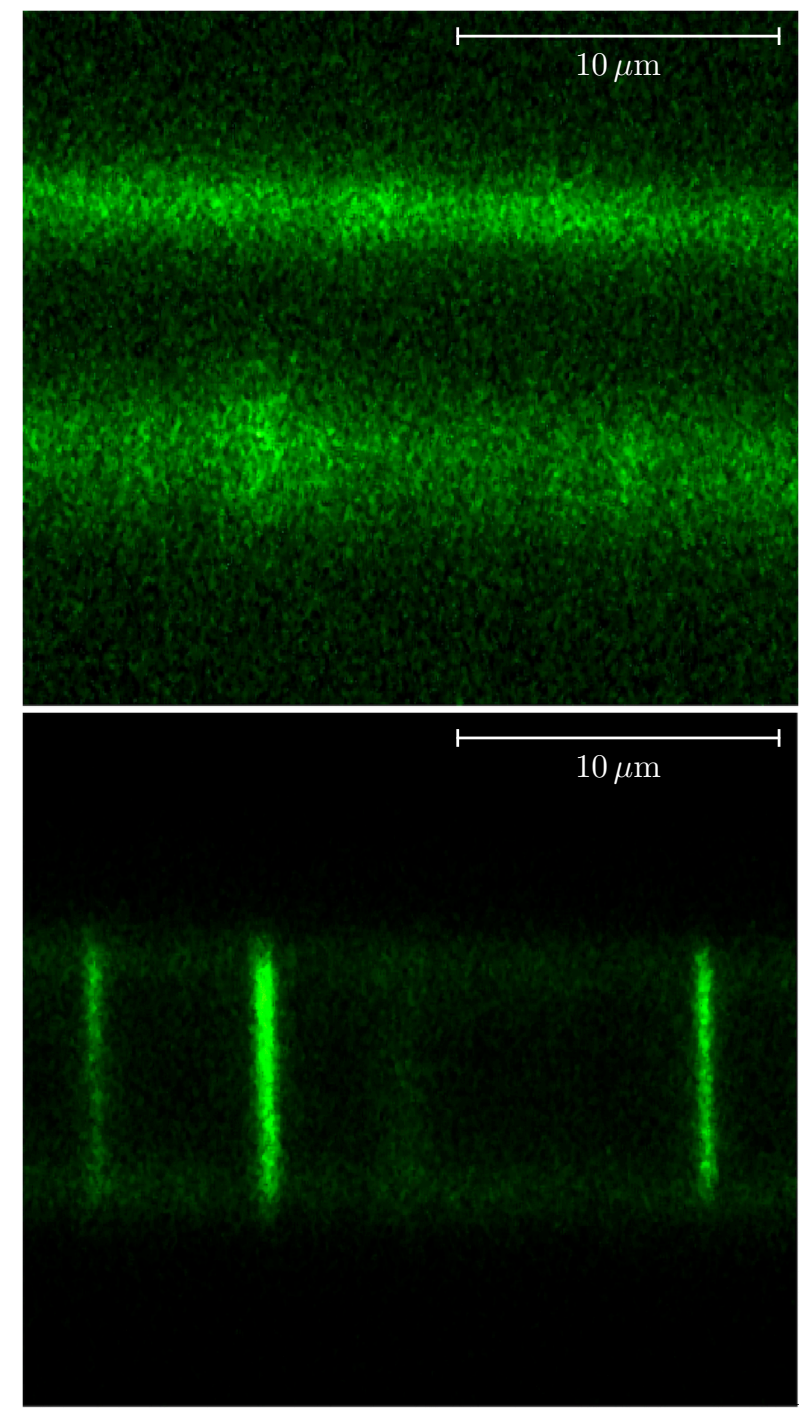

Fig. 10. CLSM images of labeled PVDF-g-PAA films modified with ethylenediamine before grafting. Images are $x z$-plan (cross-section) reconstructions of series of $x y$ slices. $z$ axis has been rescaled to account for refractive index of PVDF. Top: fluorescein isothiocyanate labeling reveals amine groups, i.e. surface oxydation. Bottom: Alexa Fluor ${ }^{\circledR}$ hydrazide labeling reveals carboxyl group, i.e. poly(acrylicacid).

Alexa Fluor ${ }^{\circledR}$ hydrazide labeling. The corresponding CLSM image is shown in Fig.10 bottom. These images show that radiografting of polyacrylic acid is selectively achieved inside membrane nanopores.

\section{Conclusion}

This paper is concerned with poly(acrylic acid) radiografting on track-etched, $9 \mu \mathrm{m}$ thick, $\beta$-PVDF membranes. Pores diameter lies in the range 20-
$50 \mathrm{~nm}$ depending on etching time, as measured by Small Angle Neutron Scattering. Our key results is to quantify that radicals produced by heavy ion irradiation still remain in PVDF films after track etching. These radicals are radially distributed along ion-tracks and allows us to specifically functionalize pore-walls by poly(acrylic acid) radiografting. This specific functionalization was evidenced for the first time by fluorescence labeling and Confocal Laser Scanning Microscopy. Membranes so functionalized have many potential applications: hydrophilic pores for aqueous solution applications (such as biological applications), single pore visualization for nanofluidic and nanofiltration physics.

\section{References}

[1] P. Apel, Swift ion effects in polymers: industrial applications., Nuclear Instr. Meth. Phys. Res. B 208 (2003) 11-20.

[2] N. Shtanko, V. Kabanov, P. Apel, M. Yoshida, The use of radiation-induced graft polymerization for modification of polymer track membranes., Nuclear Instr. Meth. Phys. Res. B 151 (1999) 416-422.

[3] R. Mazzei, E. Smolko, D. Tadey, L. Gizzi, Radiation grafting of nipaam on pvdf nuclear track membranes, Nuclear Instr. Meth. Phys. Res. B 170 (2000) 419-426.

[4] P. Wang, K. L. Tan, E. T. Kang, K. G. Neoh, Plasma-induced immobilization of poly(ethylene glycol) onto poly(vinylidene fluoride) microporous membrane, Journal of Membrane Science 195 (2002) 103-114.

[5] F. Liu, B.-K. Zhu, Y.-Y. Xu, Improving the hydrophilicity of poly(vinylidene fluoride) porous membranes by electron beam initiated surface grafting of aa/sss binary monomers, Applied Surface Science In Press, Corrected Proof.

[6] P. H. Paul, M. G. Garguilo, D. J. Rakestraw, Imaging of pressure and electrokinetically driven flows through open capillaries., Anal. Chem. 70 (1998) 2459-2467.

[7] A. Brûlet, D. Lairez, A. Lapp, J. P. Cotton, Improvement of data treatment in sans., accepted for publication in J. Appl. Cryst.

[8] A. Guinier, G. Fournet, Small-angle scattering of Xrays., J. Wiley \& sons, 1955.

[9] D. Lairez, Résolution d'un spectromètre de diffusion de neutrons aux petits angles., J. Physique IV France 9 (1999) 67-81.

[10] E. Petersohn, Ph.D. thesis, University Pierre et Marie Curie, Paris VI, France (1995).

[11] N. Betz, E. Petersohn, A. Le Moel, Swift heavy ions effects in fluoropolymers: radicals and crosslinking, Nuclear Instr. Meth. Phys. Res. B 116 (1996) 207-211.

[12] R. Spohr, Ions Tracks and Microtechnology: Principles and Application., Friedr. Vieweg \& Sohn Verlegsgschaft mbH, Braunschweig, 1990.

[13] M. C. Clochard, J. Begue, A. Lafon, D. Caldemaison, C. Bittencourt, J. J. Pireaux, N. Betz, Tailoring bulk 
and surface grafting of poly(acrylic acid) in electronirradiated pvdf, Polymer 45 (2004) 8683-8694. 